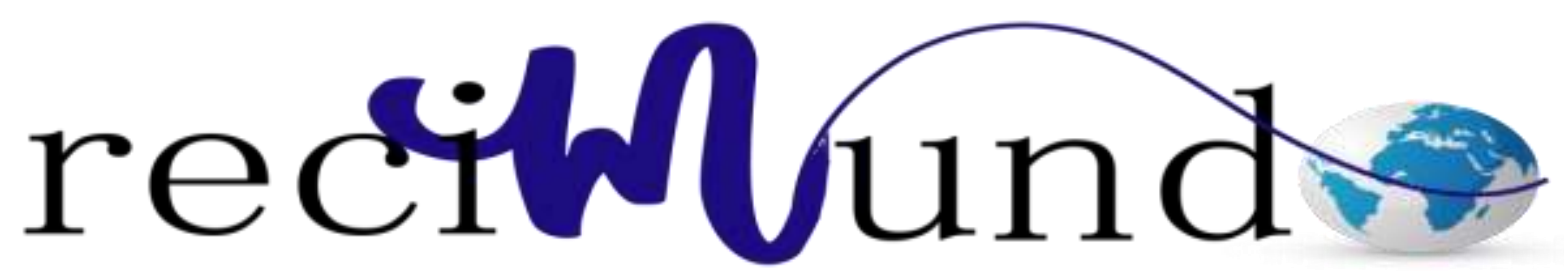

Revista Cientifica Mundo de la Investigación y el Conocimiento

\begin{abstract}
Wladimir Alberto Delgado Conforme ${ }^{\text {a; }}$ Jonathan Javier Abarca López ${ }^{\text {b }}$; Luis
\end{abstract}

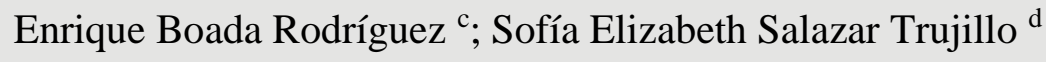

Lumbalgia inespecífica. Dolencia más común de lo que se cree

Non-specific low back pain. Most common ailment of what is believed

Revista Científica Mundo de la Investigación y el Conocimiento. Vol. 3 núm.2, abril, ISSN: 2588-073X, 2019, pp. 3-25

DOI: $\underline{10.26820 / \text { recimundo/3.(2).abril.2019.3-25 }}$

URL: http://www.recimundo.com/index.php/es/article/view/458

Código UNESCO: 3205.02 Endocrinología

Tipo de Investigación: Artículo de Revisión

Editorial Saberes del Conocimiento

Recibido: 15/01/2019

Aceptado: 18/02/2019

Publicado: 30/04/2019

Correspondencia: wladimiroconford@hotmail.com
a. Médico.
b. Médico.
c. Médico.
d. Médico. 


\title{
Lumbalgia inespecífica. Dolencia más común de lo que se cree
}

Vol. 3, núm. 2., (2019)

Wladimir Alberto Delgado Conforme; Jonathan Javier Abarca López; Luis Enrique Boada Rodríguez; Sofía Elizabeth Salazar Trujillo

\section{RESUMEN}

Múltiples estudios han demostrado que cerca del $90 \%$ de los casos de lumbalgia no presentan algún tipo de lesión demostrable, razón ésta por la que se cataloga como inespecífica. Independientemente de la manera que ocurra, la lumbalgia puede dificultar muchas de las actividades cotidianas. La metodología responde a un modelo de investigación documental para el desarrollo de una revisión de bibliografía científica, mediante la cual pudo consultarse bases de datos tales como: Intramed, Redalyc, Imbiomed, SciELO, Dialnet, Medlineplus, entre otras. El objetivo de en este trabajo radicó en exponer sobre la lumbalgia inespecífica, síntomas, clasificación, origen, factores de riesgo y su tratamiento particular, todo ello a fines de que dicho aporte sirva como herramienta de concienciación y prevención respecto a este trascendente tema. Se concluye que la lumbalgia inespecífica puede ser tan leve como un dolor ocasional o tan serio como una enfermedad específica claramente diagnosticada, así pues, parece estar ligada a una gran variedad de elementos, tales como factores fisiológicos, biomecánicos, ambientales, genéticos, sociales, organizacionales, físicos e inclusive psicológicos.

Palabras claves: Lesión; Dolor; Inespecífica; Síntomas; Riesgo.

\begin{abstract}
Multiple studies have shown that about $90 \%$ of cases of low back pain do not show any type of demonstrable injury, which is why it is classified as nonspecific. Regardless of the manner in which it occurs, low back pain can make many daily activities difficult. The methodology responds to a model of documentary research for the development of a review of scientific literature, through which databases could be consulted such as: Intramed, Redalyc, Imbiomed, SciELO, Dialnet, Medlineplus, among others. The objective of this work was to expose about non-specific low back pain, symptoms, classification, origin, risk factors and their particular treatment, all in order to make this contribution serve as a tool of awareness and prevention regarding this transcendent topic. It is concluded that non-specific low back pain can be as mild as an occasional pain or as serious as a specific disease clearly diagnosed, thus, it seems to be linked to a great variety of elements, such as physiological, biomechanical, environmental, genetic, social factors, organizational, physical and even psychological.
\end{abstract}

Key Words: Injury; Pain; Non-Specific; Symptoms; Risk. 


\section{Lumbalgia inespecífica. Dolencia más común de lo que se cree}

Vol. 3, núm. 2., (2019)

Wladimir Alberto Delgado Conforme; Jonathan Javier Abarca López; Luis Enrique Boada Rodríguez; Sofía Elizabeth Salazar Trujillo

\section{Introducción.}

Chavarría (2014) ha afirmado que la lumbalgia es padecida tanto por hombres como a mujeres de cualesquie franja etaria. Afecta de entre un 15 y $20 \%$ de la población adulta y es inespecífica en el $90 \%$ de los casos. Las personas con edades entre los 30 y 50 años son más propensas y la prevalencia de este padecimiento se vincula proporcionalmente con el aumento de la edad. (Chavarría, 2014)

En el mismo orden de ideas Hartvigsen et al. (2018) aseguran que la prevalencia puntual del dolor lumbar limitante de la actividad fue 7,3\% en 2015, lo que en líneas generales implica que, 540 millones de personas se vieron afectadas en cualquier momento. Así mismo dicen que la mayoría de las lumbalgias se denominan no específicas ya que es raro poder identificar una causa específica de dolor lumbar. Estas se caracterizan por un rango de aspectos biofísicos, psicológicos y sociales que afectan la función, la participación social y la prosperidad financiera personal. (Hartvigsen et al.; 2018)

Por su parte, Vlaeyen et al. (2018), igualmente afirma que "La gran mayoría de los pacientes con dolor de espalda tienen dolor inespecífico, por lo que no se ha identificado una patología subyacente o un contribuyente nociceptivo". En cuanto al aspecto epidemiológico se fundamenta en el reciente Estudio de la Carga Mundial de Enfermedad de 2016, para afirmar concisamente que el dolor lumbar "es una carga sustancial para los pacientes y la sociedad", ya que a través del mismo se ha evidenciado que, en los 188 países evaluados, figuró una alta prevalencia del dolor lumbar como una las diez (10) causas primordiales por las cuales las personas vivieron años con discapacidad. Aparte, agregan un importante dato sobre América 


\section{Lumbalgia inespecífica. Dolencia más común de lo que se cree}

Vol. 3, núm. 2., (2019)

Wladimir Alberto Delgado Conforme; Jonathan Javier Abarca López; Luis Enrique Boada Rodríguez; Sofía Elizabeth Salazar Trujillo

Latina al referir que, de acuerdo a una revisión sistemática, se ha estimado una prevalencia del 31,3\% respecto al punto medio de dolor lumbar crónico. (Vlaeyen et al.; 2018)

El objetivo de en este trabajo radica en exponer sobre la lumbalgia inespecífica, síntomas, clasificación, origen, factores de riesgo y su tratamiento particular, todo ello a fines de que dicho conocimiento; basado en la literatura científica vigente y disponible en las distintas bases de datos consultadas, sirva como herramienta de concienciación y prevención respecto a este trascendente tema.

\section{Metodología.}

Para el desarrollo de la presente revisión, se ha propuesto un modelo de investigación documental, y para tal fin se ha considerado realizar la búsqueda de información mediante el uso de algunas bases de datos, tales como: Intramed, Redalyc, Imbiomed, SciELO, Dialnet, Medlineplus, entre otras. El criterio de búsqueda se delimitó con las expresiones: "Lumbalgia", "Lumbalgia inespecífica" y "Dolor lumbar", obteniendo como resultado un cúmulo de material identificado como títulos, citas y referencias asociadas a artículos científicos de investigación, casos clínicos, revistas especializadas, libros, boletines, folletos, tesis, datos estadísticos, notas informativas, publicaciones formales y demás recursos bibliográficos, que sucesivamente fueron filtrados considerando ahora criterios de idioma español e inglés y vigencia en los últimos diez años. Finalmente se escogen al azar poco más de treinta títulos relevantes y más vinculantes al tema central, procurando siempre mantener el principio de mayor vigencia, los cuales fueron totalmente leídos, analizados, interpretados y discutidos por el equipo investigador, siendo así que paulatinamente se fue fundamentando científicamente las bases teóricas, ideas y argumentos 


\section{Lumbalgia inespecífica. Dolencia más común de lo que se cree}

Vol. 3, núm. 2., (2019)

Wladimir Alberto Delgado Conforme; Jonathan Javier Abarca López; Luis Enrique Boada Rodríguez; Sofía Elizabeth Salazar Trujillo

plasmados como resultados de este compendio, lo que a su vez facilitó la redacción de la conclusión aquí alcanzada.

Ahora bien, es idóneo el momento para conceptualizar lo que se entiende por investigación documental, y para ello se hace referencia al aporte de Alfonso (1995) quien citado en el trabajo de Morales (2003) indica que "es un procedimiento científico, un proceso sistemático de indagación, recolección, organización, análisis e interpretación de información o datos en torno a un determinado tema. Al igual que otros tipos de investigación, éste es conducente a la construcción de conocimientos. (Morales, 2003).

Por su parte , Morales (2003), tras su discernimiento no pierde la oportunidad de explicar que la investigación documental "tiene la particularidad de utilizar como una fuente primaria de insumos, mas no la única y exclusiva, el documento escrito en sus diferentes formas" entre los que se encuentran los documentos impresos y electrónicos, tales como: libros enciclopedias, revistas, periódicos, diccionarios, monografías, tesis y otros documentos, correos electrónicos, CD Roms, bases de datos y páginas Web; y los recursos audiovisuales, que vendrían siendo: mapas, fotografías, ilustraciones, videos, programas de radio y de televisión, canciones, grabaciones y otros tipos de medios de información. Finalmente indica que:

Como en el proceso de investigación documental se dispone, esencialmente, de documentos; que son el resultado de otras investigaciones, de reflexiones de teóricos, lo cual representa la base teórica del área objeto de investigación; el conocimiento se construye a partir de su lectura, análisis, reflexión e interpretación de dichos documentos. (Morales, 2003) 


\section{Lumbalgia inespecífica. Dolencia más común de lo que se cree \\ Vol. 3, núm. 2., (2019) \\ Wladimir Alberto Delgado Conforme; Jonathan Javier Abarca López; Luis Enrique Boada Rodríguez; Sofía Elizabeth Salazar Trujillo}

\section{Resultados.}

El término lumbalgia o lumbago se define como todo aquel dolor común de duración variable, circunscrito a la parte baja de la espalda o zona lumbar. Éste suele ser intenso y profundo, teniendo como efecto final una repercusión en la movilidad normal de la zona, debido a la sensación dolorosa. En cuanto a la percepción del dolor, se apoyan en otros tratadistas para explicar que es subjetivo, ya que éste pudiera percibirse de manera muy distinta entre uno y otra persona, y es porque pudieran encontrarse pacientes que, con mínimas lesiones, relatan un dolor insoportable, y otros con enfermedades graves e incluso mortales que, inicialmente, presentan síntomas dolorosos de escasa intensidad (Aguilera \& Herrera; 2013 p. 80-81)

Para definir la lumbalgia desde el punto de vista clínico, es valioso extraer del aporte de Chavarría (2014), quien apoyándose en la idea de otros expertos, ha dicho que es aquel dolor que ocurre entre el margen inferior de las últimas costillas (es decir, de la parrilla costal) hasta la región glútea inferior (o lo que sería igualmente, la zona ubicada por encima de las líneas glúteas inferiores), pudiendo ocurrir o no, dolor en los miembros inferiores; a su vez, este dolor es, generalmente, acompañado de espasmo, que compromete las estructuras osteomusculares y ligamentarias del raquis y su etiología es múltiple. (Chavarría, 2014)

Del trabajo de Vlaeyen et al. (2018), se puede extraer y a la vez comprender que, sin distingo de edad y género, muchas personas padecerán de dolor de espalda (dolor lumbar) al menos una vez en la vida, siendo de allí su alta incidencia. Así mimo ha explicado que la lumbalgia se concibe como "una condición compleja y multifactorial que probablemente se desarrolla como resultado de la interacción entre varios factores de riesgo". Apoyándose en otros 


\section{Lumbalgia inespecífica. Dolencia más común de lo que se cree}

Vol. 3, núm. 2., (2019)

Wladimir Alberto Delgado Conforme; Jonathan Javier Abarca López; Luis Enrique Boada Rodríguez; Sofía Elizabeth Salazar Trujillo

tratadistas continúa complementando su argumentación al referir que "La gran mayoría de los pacientes con dolor de espalda tienen dolor inespecífico, por lo que no se ha identificado una patología subyacente o un contribuyente nociceptivo" (Vlaeyen et al.; 2018)

Ahlers et al. (2010) citado por Pérez (2018) ha definido la lumbalgia como: "presencia de malestar en la zona lumbar, localizado entre el borde inferior de las últimas costillas y el pliegue inferior de la zona glútea, con o sin irradiación a una o ambas piernas, compromete estructuras osteomusculares y ligamentarias, con o sin limitación funcional que dificultan las actividades de la vida diaria y que puede causar ausentismo laboral” (Pérez; 2018; p. 11)

Igualmente, Martínez (2015) se fundamenta en el aporte de otros tratadistas para explicar que:

La lumbalgia se define como dolor, tensión o rigidez localizada por debajo del margen costal posterior y por arriba de los pliegues glúteos con o sin dolor referido a la extremidad inferior. Puede originarse en diversas estructuras espinales: ligamentos, facetas articulares, periostio vertebral, musculatura y fascias paravertebrales, anillo fibroso y/o raíces nerviosas. La etiología más común está constituida por las lesiones músculo-ligamentosas y procesos degenerativos tanto del disco intervertebral como de las facetas articulares. Sin embargo, hasta en $85 \%$ de los pacientes no es posible definir el origen anatomopatológico durante el episodio agudo de dolor. La Organización Mundial de la Salud (OMS) señala que el dolor de espalda baja no es ni una enfermedad ni una entidad diagnóstica, sino que se trata del dolor de duración variable en un área de la anatomía, afectada de manera tan frecuente que se ha convertido en un paradigma de respuestas a estímulos externos e internos. 


\section{Lumbalgia inespecífica. Dolencia más común de lo que se cree}

Vol. 3, núm. 2., (2019)

Wladimir Alberto Delgado Conforme; Jonathan Javier Abarca López; Luis Enrique Boada Rodríguez; Sofía Elizabeth Salazar Trujillo

Es sabido que el cuadro original de lumbalgia es de inicio agudo, mientras que la recurrencia ulterior tiende a aparecer en forma insidiosa. El dolor crónico de espalda es una condición más difícil, ya que presenta una intrincada cubierta psicológica por la insatisfacción laboral, aburrimiento y un sistema de compensación generoso que contribuye a ello. Se debe tener en cuenta que un porcentaje de casos con esta patología está asociado a neoplasias, infecciones o patologías en órganos adyacentes. Los estudios experimentales sugieren que el dolor de espalda baja se puede originar en muchas estructuras de la columna, incluyendo ligamentos, facetas articulares, periostio vertebral, musculatura paravertebral y fascias, vasos sanguíneos, el annulus fibroso y las raíces nerviosas. Posiblemente el dolor de espalda baja sea causado por lesiones músculo-ligamentarias y procesos degenerativos relacionados con la edad en el disco intervertebral y en las facetas articulares1 (Martínez; 2015; p. 8-10)

\section{Lumbalgia inespecífica}

Balagué, Mannion, \& Pellisé (2012) la definen como el “dolor lumbar no atribuible a una patología específica, como por ejemplo: infección, tumor, osteoporosis, fractura, deformidad estructural, trastorno inflamatorio, síndrome radicular o síndrome de la cola de caballo"

\section{Determinación de la lumbalgia inespecífica}

Pérez et al. (2013) básicamente dice que puede ser asumida en ausencia de las señales de alerta propias de una enfermedad sistémica o las inherentes para la derivación a cirugía, que en resumen serían:

1. Dolor en niños o adolescentes $<20$ años (ó $>55$ años) que aparece por primera vez. 


\section{Lumbalgia inespecífica. Dolencia más común de lo que se cree}

Vol. 3, núm. 2., (2019)

Wladimir Alberto Delgado Conforme; Jonathan Javier Abarca López; Luis Enrique Boada Rodríguez; Sofía Elizabeth Salazar Trujillo

2. Antecedente de trauma violento.

3. Dolor nocturno.

4. Antecedente de neoplasia.

5. Esteroides sistémicos.

6. Drogadicción y/o HIV.

7. Pérdida de peso.

8. Sensación de enfermedad.

9. Movilidad muy disminuida.

10. Deformidad evidente.

11. Trastornos esfínteres.

12. Pérdida progresiva de fuerza.

13. Espondilitis.

14. Rigidez matutina

15. Afectación de otras articulaciones.

16. Iritis, colitis, uretritis

17. Fiebre.

18. Soplo abdominal

\section{Síntomas}

De acuerdo a la publicación de la Academia Americana de Cirujanos Ortopédicos (AAOS, por sus siglas en inglés) (2009) se comprende que la sintomatología de la lumbalgia es variable, por lo que puede ser intenso o punzante, también pudiera ser un dolor sordo, fijo o sentirse como un espasmo muscular. Aseguran que la percepción del dolor dependerá de la causa subyacente de la lumbalgia. En la mayoría de los casos, independientemente de la causa subyacente, el dolor por la lumbalgia mejora al reclinarse o recostarse.

Las personas que sufren de lumbalgia pueden experimentar alguno de lo siguiente: 


\section{Lumbalgia inespecífica. Dolencia más común de lo que se cree}

Vol. 3, núm. 2., (2019)

Wladimir Alberto Delgado Conforme; Jonathan Javier Abarca López; Luis Enrique Boada Rodríguez; Sofía Elizabeth Salazar Trujillo

- La lumbalgia se intensifica al inclinarse hacia delante o al levantar un objeto.

- Permanecer sentado puede agravar el dolor.

- Estar de pie o caminar puede agravar el dolor.

- El dolor de espalda va y viene, y por lo general sigue un curso de subidas y bajadas con días buenos y malos.

- El dolor puede extenderse de la espalda a la nalga o parte exterior de la cadera, sin extenderse hacia la pierna.

- La ciática es común con un disco herniado. Esto incluye dolor en la nalga y pierna y hasta entumecimiento, cosquilleo, o debilidad que continúa hacia el pie. Es posible padecer de ciática sin tener dolor de espalda.

Refieren que, sin distinción de edad o síntomas, si en unas pocas semanas el dolor de espalda no mejora, o si éste se produce a la par de una fiebre, escalofríos o pérdida de peso imprevista, el deber es ir a consultar con un médico. (AAOS, 2009)

\section{Clasificación}

En atención primaria, el dolor lumbar se puede clasificar en una de cuatro categorías: un problema más allá de la columna lumbar (por ejemplo, cálculos renales); un trastorno grave que afecta la columna lumbar (por ejemplo, absceso epidural); dolor lumbar que se presenta con dolor radicular (por ejemplo, relacionado con una hernia de disco intervertebral) o claudicación neurogénica (por ejemplo, relacionado con una estenosis del canal espinal central); o dolor lumbar inespecífico. (Traeger, Buchbinder, Harris, \& Maher, 2017) 


\section{Lumbalgia inespecífica. Dolencia más común de lo que se cree}

Vol. 3, núm. 2., (2019)

Wladimir Alberto Delgado Conforme; Jonathan Javier Abarca López; Luis Enrique Boada Rodríguez; Sofía Elizabeth Salazar Trujillo

Origen (Etiopatogenia)

De acuerdo al trabajo de Pérez et al. (2013) se logra comprender que en el $90 \%$ de los casos de lumbalgia se deben a causas vertebrales y paravertebrales, no obstante, otros tantos casos tienen un origen multifactorial, subsecuentes de la existencia de múltiples procesos y de allí la dificultad para identificar la causa originaria. Así mismo, el 10\% de dichos casos suelen cronificarse por lo que repercuten significativamente a nivel personal, familiar, laboral y económico. Enfatizan que en el $80 \%$ de los casos, los hallazgos son inespecíficos y tan solo en un $20 \%$ de los mismos puede determinarse la causa etiológica, de estos entre un 3-5\% presentan una patología subyacente grave.

Complementan su exposición facilitando una clasificación etiopatogénica del dolor lumbar, sin embargo, aclaran que se hace necesario verificar "unas consideraciones generales aportadas por el grupo de Québec" que en resumen se refieren a la conclusión de que:

1. En la mayoría de los pacientes no se encuentra una alteración estructural.

2. La mayoría son autolimitadas en el tiempo y de curso benigno.

3. Las exploraciones diagnósticas producen escaso beneficio, siendo la descripción del dolor relatada por el paciente y su localización la aportación más valiosa. (Pérez et al.; 2013; p. 741-742)

\section{Clasificación etiopatogénica del dolor lumbar}

\section{Lumbalgia mecánica}

1.1. Alteraciones estructurales

1.1.1. Discal 


\section{Lumbalgia inespecífica. Dolencia más común de lo que se cree}

Vol. 3, núm. 2., (2019)

Wladimir Alberto Delgado Conforme; Jonathan Javier Abarca López; Luis Enrique Boada Rodríguez; Sofía Elizabeth Salazar Trujillo

\subsubsection{No irradiado}

1.1.1.2. Irradiado

1.1.2. Facetario

1.1.3. Estenosis de canal

1.1.4. Espondilólisis y espondilolistesis

1.1.5. Escoliosis

1.1.6. Síndrome de la Cauda equina

1.2. Sobrecarga funcional y postural

2. Lumbalgia no mecánica

2.1. Inflamatoria: Espondiloartropatías

2.2. Infecciosa

2.2.1. Bacterias

2.2.2. Micobacterias

2.2.3. Hongos

2.2.4. Espiroquetas

2.2.5. Parásitos

2.3. Tumoral

2.3.1. Tumores óseos benignos

2.3.2. Tumores óseos malignos

2.3.3. Metástasis vertebrales

2.3.4. Tumores intra raquídeos

2.4. Otras causas

2.4.1. Enfermedades endocrinas y metabólicas

2.4.2. Enfermedades hematológicas

2.4.3. Miscelánea:

- Enfermedad de Paget

- Sarcoidosis vertebral

- Enfermedad de Charcot

- Enfermedades hereditarias

3. Dolor psicosomático

3.1. Síndromes miofasciales

3.2. Fibromialgia

De la misma manera Ique \& Nogueira (2017) han descrito la etiopatogenia de esta condición de la siguiente manera: 


\section{Lumbalgia inespecífica. Dolencia más común de lo que se cree}

Vol. 3, núm. 2., (2019)

Wladimir Alberto Delgado Conforme; Jonathan Javier Abarca López; Luis Enrique Boada Rodríguez; Sofía Elizabeth Salazar Trujillo

Dolor lumbar no radicular: tiene origen mecánico (fuerzas excesivas), postural (posiciones viciosas de la columna), funcional (movimientos inadecuados de la columna) o muscular y se asocia al mal estado físico, al sobrepeso y al sobresfuerzo.

Las lumbalgias sin irradiación pueden ser manifestaciones de trastornos de somatización o de depresión.

Torceduras y lesiones: lesiones leves y de evolución limitada, asociadas al levantamiento de objetos, caídas, desaceleraciones bruscas como en los accidentes de tránsito. Por lo general no hay una lesión anatómica específica.

Fracturas vertebrales: la mayoría se debe a lesiones por compresión o flexión que producen una cuña o compresión anterior, en traumas más graves puede haber fracturas, luxaciones y fracturas por estallido y con frecuencia se deben a precipitaciones y lesiones directas.

Dolor lumbar radicular: Compresión mecánica, mecanismos microvasculares o histoquímicos del disco que pueden alterar la fisiología de la raíz nerviosa. Patologías involucradas:

\section{Enfermedad discal:}

- Protrusión discal: el anillo del disco intervertebral está intacto, pero engrosado o abultado. 


\section{Lumbalgia inespecífica. Dolencia más común de lo que se cree}

Vol. 3, núm. 2., (2019)

Wladimir Alberto Delgado Conforme; Jonathan Javier Abarca López; Luis Enrique Boada Rodríguez; Sofía Elizabeth Salazar Trujillo

- Extrusión discal: el núcleo pulposo ha penetrado el anillo fibroso y puede alojarse debajo del ligamento longitudinal posterior o romperlo.

- Disco secuestrado: el material nuclear ha roto su contención en el anillo y ligamento y los fragmentos libres entran en contacto con la raíz nerviosa. El material secuestrado ocasionalmente migra a otros niveles, superior o inferior del disco inicialmente comprometido. La discopatía crónica puede deberse a osteocondrosis, engrosamiento del anillo fibroso, esclerosis ósea, osteofitosis y estrechamiento del foramen intervertebral.

Espondilolisis (defecto óseo en la porción interarticular por defecto congénito o posterior a fracturas, el defecto que en general es bilateral se observa en las proyecciones oblicuas de las radiografías y se asocia a traumatismos únicos, lesiones leves repetidas o el crecimiento) y Espondilolistesis (desplazamiento de una vértebra lumbar sobre su vecina inferior, más frecuente en L5- S1). Es difícil diferenciar una espondilosis o espondilolistesis congénita de una traumática en una radiografía, es importante para hacer la diferenciación la historia brindada, la clínica y el mecanismo de trauma que describe, la espondilólisis congénita es muy rara. Si en la radiografía se observa una columna con cambios degenerativos y una listesis probablemente se deba a la degeneración de la misma, en cambio si la columna nopresenta cambios degenerativos y unalistesis probablemente es por trauma.

Espina bífida: La espina bífida es el defecto congénito discapacitante más común en los Estados Unidos. Es un tipo de defecto del tubo neural que constituye un problema en la médula espinal o las membranas que la recubren. En la espina bífida, la columna vertebral no se cierra 


\section{Lumbalgia inespecífica. Dolencia más común de lo que se cree}

Vol. 3, núm. 2., (2019)

Wladimir Alberto Delgado Conforme; Jonathan Javier Abarca López; Luis Enrique Boada Rodríguez; Sofía Elizabeth Salazar Trujillo

completamente durante el primer mes de embarazo y puede cursar asintomática por mucho tiempo.

Espondiloartropatías inflamatorias, como la espondilitis anquilosante, que aparece entre los 20 y los 40 años de edad, con dolor y rigidez dorsolumbar progresiva. La molestia tiene predominio matutino y los hallazgos característicos en la radiología son tardíos. Otras espondiloatropatías también se pueden asociar con la psoriasis y el Síndrome de Reiter.

Otras patologías: tumores óseos, dolor irradiado desde las vísceras, lesiones neoplásicas neurales o infecciones entre otros. Los pacientes con osteoporosis y otras enfermedades metabólicas óseas, pueden tener colapsos vertebrales dolorosos. En pacientes ancianos, con o sin dolor lumbar importante, se observan en los estudios radiológicos cambios de envejecimiento o espondilosis (enfermedad degenerativa, se presenta en edades avanzadas de la vida y los cambios radiológicos no siempre corresponden con la clínica).

El espectro de la lesión degenerativa incluye el estrechamiento de los espacios discales en uno o varios niveles, imágenes artrósicas en las facetas articulares y compromiso variable de estenosis del foramen intervertebral y desviaciones de la columna.

Las causas de dolor recurrente son: mecánicas (hernia discal, fibrosis posquirúrgica, inestabilidad espinal o estenosis lumbar) y no mecánicas (aracnoiditis y discitis (ambas pueden darse posterior a cirugías, estudios con medios de contraste o traumáticos) inestabilidad psicosocial y enfermedades sistémicas). (Ique \& Nogueira; 2017; p. 20-22) 


\section{Lumbalgia inespecífica. Dolencia más común de lo que se cree}

Vol. 3, núm. 2., (2019)

Wladimir Alberto Delgado Conforme; Jonathan Javier Abarca López; Luis Enrique Boada Rodríguez; Sofía Elizabeth Salazar Trujillo

Factores de riesgo

Pérez (2018) basándose en los resultados obtenidos de otros estudios, ha sintetizado algunos de los factores más relevantes asociados a este padecimiento, siendo así que se extrae lo siguiente:

Sexo

Los estudios realizados en cuanto a esta variable de estudio tienen diversos resultados en los unos indican que las mujeres son las más afectadas y en otros al contrario los hombres. Algunos autores de estudios realizados informan que las edades más activas laboralmente tanto los hombres y mujeres tienen, la misma frecuencia de lumbalgia. El investigador Hestbaek precisa que son las mujeres quienes asumen una mayor prevalencia de dolor lumbar en comparación con los hombres

\section{Edad}

La edad comprendida entre los 20 y 40 años suele ser las más frecuentes para el debut de esta enfermedad primera presentación de la lumbalgia siendo los 30 años la edad más común. En otras revisiones se encontró que los grupos de edades afectadas tenemos que es la principal causa de limitación de actividades en los menores de 45 años y la tercera en las personas mayores de 45 años, siendo así la patología musculo esquelética de mayor prevalencia en los mayores de 65 años. 


\section{Lumbalgia inespecífica. Dolencia más común de lo que se cree}

Vol. 3, núm. 2., (2019)

Wladimir Alberto Delgado Conforme; Jonathan Javier Abarca López; Luis Enrique Boada Rodríguez; Sofía Elizabeth Salazar Trujillo

Talla

En un estudio realizado en los trabajadores industriales, se encontró que los de estatura más alta es decir igual o superior a $178 \mathrm{~cm}$, presentaron lumbalgia en menos frecuencia que los trabajadores de talla media comprendida entre los 166 y $177 \mathrm{~cm}$, las personas con estatura más baja igual o inferior a $164 \mathrm{~cm}$, tuvieron dolor lumbar con la misma frecuencia que los de estatura media. En otros estudios también se encontró que la talla tiene relación a casos de lumbalgia es así que se determinó que tener una estatura más alta es mayor riesgo de lumbalgia.

Peso e índice de masa corporal (IMC)

De acuerdo con varias investigaciones realizadas se ha verificado que existe una clara asociación entre problemas de peso como lo es el sobrepeso y dolor lumbar ya sea en los eventos que se presenta con mayor frecuencia o con un mayor número de episodios si no se tuviese sobrepeso, además si no se logra controlar el peso, lo más probable es que al padecer lumbalgia se termine en un proceso crónico. [...] En un estudio para determinar si existe o no asociación entre el IMC y Lumbalgia se pudo observar que esta incremento hasta en $22 \%$ por cada $\mathrm{kg} / \mathrm{m} 2$ de incremento del peso con respecto del grupo de pacientes con IMC normal. En otra revisión sobre esta variable se informó que de acuerdo al Índice de masa corporal esta patología se incrementa en 1.53 veces más el riesgo de llegar a padecer lumbalgia. En otro estudio realizado en México se determinó que la población con sobrepeso tiene un 5.7 veces más riesgo de padecer lumbalgia en comparación con la población que presenta un índice masa corporal normal. 


\section{Lumbalgia inespecífica. Dolencia más común de lo que se cree \\ Vol. 3, núm. 2., (2019) \\ Wladimir Alberto Delgado Conforme; Jonathan Javier Abarca López; Luis Enrique Boada Rodríguez; Sofía Elizabeth Salazar Trujillo}

\section{Residencia}

El lugar de residencia y esta enfermedad también se encuentran asociados ya que el vivir en un medio Rural no es igual al ambiente Urbano, debido a las distintas actividades que se realizan. En un estudio realizado en España se determinó que vivir en una zona rural representaba un factor de riesgo para lumbalgia, que de acuerdo a los expertos están dadas por las diferencias culturales a las que están expuestas las personas dependiendo de sus lugares de residencia. En el mismo estudio mencionado antes, realizado en Cuenca en el año 2014, se concluyó que las personas que vivían en el medio Rural fueron las más afectadas debido a sus actividades.

\section{Relacionados con el trabajo}

El autor Habib, determinó evidencia entre el dolor lumbar y factores laborales como los son: realizar trabajos pesados, posturas inadecuadas, flexiones o giros del tronco, los levantamientos y movimientos potentes, realizar durante largas jornadas trabajos repetitivos y las vibraciones. Al revisar otro estudio se halló que el dolor lumbar invalidante se relaciona con actividades como lo son el puesto de trabajo, la antigüedad en el puesto de trabajo, el esfuerzo físico realizado, levantamiento de cargas, cantidad de peso levantado, acciones de flexión, extensión y rotación de la espalda, indicándonos este estudio que la lumbalgia es 2,5 veces mayor en los trabajadores expuestos a esfuerzos y cargas físicas pesadas además de posiciones forzadas si se los comparaba con las personas que desempeñaban cargos administrativos, servicios técnicos y profesionales sometidos a menor demanda física [...] Existe un mayor peligro de lumbalgia en relación con el trabajo es el movimiento de torsión que se realice en más 


\section{Lumbalgia inespecífica. Dolencia más común de lo que se cree}

Vol. 3, núm. 2., (2019)

Wladimir Alberto Delgado Conforme; Jonathan Javier Abarca López; Luis Enrique Boada Rodríguez; Sofía Elizabeth Salazar Trujillo

de 20 veces ocasiones diarias y con un peso mayor a $10 \mathrm{Kg}$., y si sumamos a esto patologías asociadas como osteoporosis, espondilitis u otras patologías vertebrales la lumbalgia terminará en un problema crónico. En lo que se refiere a lumbalgia y trabajo existe una clara asociación entre los factores de riesgo como lo son: los horarios extendidos de trabajo, distintos tipos de trabajo, el uso o no de prendas de protección adecuadas. (Pérez; 2018; p. 21-25)

\section{Tratamientos}

Las recomendaciones en el tratamiento de la lumbalgia estás basadas en la evidencia científica disponible, resultado de estudios validados que determinan eficacia, efectividad o eficiencia de una técnica, por lo tanto, pueden existir tratamientos que sean efectivos, pero si no existe evidencia de la misma no se recomiendan. La lumbalgia aguda se resuelve antes de las 4 semanas en el $70 \%$ de los casos, su impacto sobre la incapacidad y la calidad de vida depende más que de su intensidad de su duración, siendo el tratamiento de la lumbalgia inespecífica multidisciplinar y no exclusiva de ninguna especialidad, debiendo existir una buena comunicación e interrelación entre los distintos profesionales. (Pérez et al.; 2013; p. 755)

Sin embargo, en relación al manejo del dolor lumbar agudo inespecífico, alegan que se ha dado un consenso en la mayoría de las guías clínicas (según la Guía de práctica clínica sobre lumbalgia Osakidetza / GPG, 2007) y las mismas, al respecto refieren que, como medidas generales se debe:

a. Informar al paciente de manera oral, positiva y tranquilizadora la importancia de mantener la actividad y el pronóstico favorable, tratando de fomentar actitudes activas y 


\section{Lumbalgia inespecífica. Dolencia más común de lo que se cree}

Vol. 3, núm. 2., (2019)

Wladimir Alberto Delgado Conforme; Jonathan Javier Abarca López; Luis Enrique Boada Rodríguez; Sofía Elizabeth Salazar Trujillo

suprimir el miedo, asegurándose de destacar, entre otras cosas, que: no existe enfermedad subyacente grave ni alteraciones estructurales irreversibles, el episodio agudo desaparece antes de las 2 semanas y tiene un buen pronóstico, realizar pruebas radiológicas resultan innecesarias. La información adecuada mejora el estado funcional del paciente, acelera su recuperación y disminuye el número de visitas.

b. Actividad y reposo. Evitar el reposo en cama y mantener el grado de actividad física que le permita el dolor (incluido el trabajo) teniendo en cuenta las preferencias y habilidades de cada persona. El reposo no es efectivo y puede retrasar la recuperación del paciente. La recomendación es continuar con las actividades habituales; sin embargo, los movimientos corporales tales como la flexión, el giro, el estiramiento y las actividades vigorosas deben ser evitadas.

c. Ejercicio terapéutico. El ejercicio y escuela de espalda no están indicados en lumbalgia aguda. Basado en la evidencia, no existe una diferencia importante en los efectos del reposo en cama comparado con la recomendación de permanecer activo en el tratamiento de la lumbalgia aguda/subaguda.

d. Control del sobrepeso. En cuanto a la obesidad y las medidas dietéticas para perder peso, no existen suficientes estudios de calidad en los que se pueda evidenciar la asociación entre el dolor lumbar y el sobrepeso, sin embargo, esto no debe suponer un cambio en el consejo sobre la conveniencia de evitar el sobrepeso. (Pérez et al.; 2013; p. 755-756) 


\section{Lumbalgia inespecífica. Dolencia más común de lo que se cree}

Vol. 3, núm. 2., (2019)

Wladimir Alberto Delgado Conforme; Jonathan Javier Abarca López; Luis Enrique Boada Rodríguez; Sofía Elizabeth Salazar Trujillo

\section{Prevención}

El grupo más complicado a la hora de la prevención es el de la lumbalgia mecánica inespecífica, que engloba a la mayor parte de los pacientes y en los cuales no puede determinarse una causa etiológica. La prevención de estos procesos será evitando todos los factores de mal pronóstico que se mencionan a continuación: A) Factores demográficos (sexo: varones, edad: laboral, nivel educativo bajo, nivel socioeconómico bajo); B) Factores laborales (trabajo mecánico: vibración, flexión, torsión; trabajo monótono y repetitivo, insatisfacción laboral, bajas laborales, litigios laborales, compensaciones económicas, accidente laboral percibido; C) Factores médicos (enfermedades concomitantes, síndrome doloroso crónico, drogodependencia o abuso de sustancias; indicadores de salud asociados: fatigabilidad y cansancio, cefaleas, alteraciones del sueño, molestias digestivas inespecíficas; sedentarismo, discapacidad asociada) y D) Factores psicológicos (antecedentes de patología psiquiátrica, depresión o ansiedad, alteraciones de la personalidad, baja capacidad de afrontamiento)

\section{Conclusión.}

La lumbalgia, asociada a los trastornos del aparato locomotor, puede ser tan leve como un dolor ocasional o tan serio como una enfermedad específica claramente diagnosticada, así pues, parece estar ligada a una gran variedad de elementos, tales como factores fisiológicos, biomecánicos, ambientales, genéticos, sociales, organizacionales, físicos e inclusive psicológicos. 


\section{Lumbalgia inespecífica. Dolencia más común de lo que se cree}

Vol. 3, núm. 2., (2019)

Wladimir Alberto Delgado Conforme; Jonathan Javier Abarca López; Luis Enrique Boada Rodríguez; Sofía Elizabeth Salazar Trujillo

Definitivamente, lo que ayudará a un médico de atención primaria en la identificación de un paciente que necesita una referencia hacia un especialista, es la elaboración de una historia clínica completa y un examen físico cuidadoso, en lugar de pruebas de laboratorio o diagnóstico por imágenes, de allí pues, bien pudiera decirse que surge la necesidad inequívoca de prestar especial atención al enfoque multidisciplinario con debe abordarse estos síntomas, porque lamentablemente es frecuente que con solo la evaluación clínica se pasen por alto los pasos clave de la identificación de enfermedades graves.

\section{Bibliografía.}

Aguilera, A., \& Herrera, A. (julio-diciembre de 2013). Lumbalgía: Una dodelncia muy popular y a la vez desconocida. Comunidad y Salud, 11(2), 80-89. Obtenido de https://www.redalyc.org/articulo.oa?id=375740253010

Balagué, F., Mannion, A., \& Pellisé, F. (04 de 02 de 2012). Non-specific low back pain. Lancet, 379(9814), 482-491. doi:https://doi.org/10.1016/S0140-6736(11)60610-7

Chavarría, J. (2014). Lumbalgia: Causas, Diagnóstico y Manejo. Revista Médica de Costa Rica y Centroamérica, 71(611), 447-454. Obtenido de http://www.medigraphic.com/pdfs/revmedcoscen/rmc-2014/rmc143n.pdf

Hartvigsen, J., Hancock, M., Kongsted, A., Louw, Q., Ferreira, M., \& Genevay, S. (09 de 06 de 2018). Low Back Pain. The Lancet, 391(10137), 2356-2367. doi:https://doi.org/10.1016/S0140-6736(18)30480-X

Ique, B., \& Nogueira, C. (2017). Incidencia, factores de riesgo y prevención de lumbalgia por método de pilates en dos asociaciones de mototaxistas de Iquitos, 2016. Universidad Cietífica del Perú, Facultad de Ciencias de la Salud. Iquitos: Universidad Cietífica del Perú. Obtenido de http://repositorio.ucp.edu.pe/bitstream/handle/UCP/277/IQUENOGUEIRA-1-Trabajo-Incidencia.pdf?sequence=1\&isAllowed=y

Martínez, O. (2015). Calidad de Vida en pacintes con Lumbalgia. Veracruz - México: Universidad Veracruzana. Obtenido de https://cdigital.uv.mx/bitstream/handle/123456789/42162/MartinezGarciaOmar.pdf?sequ ence $=1 \&$ isAllowed $=\mathrm{y}$ 


\section{Lumbalgia inespecífica. Dolencia más común de lo que se cree}

Vol. 3, núm. 2., (2019)

Wladimir Alberto Delgado Conforme; Jonathan Javier Abarca López; Luis Enrique Boada Rodríguez; Sofía Elizabeth Salazar Trujillo

Morales, O. (2003). Fundamentos de la Investigación Documental y la Monografía. En F. d. Grupo Multidisciplinario de Investigación en Odontología, \& N. E. Rincón (Ed.), Manual para la elaboración y presentación de la monografía (pág. 20). Merida, Merida, Venezuela: Universidad de Los Andes (ULA). Obtenido de www.saber.ula.ve/bitstream/handle/123456789/16490/fundamentos_investigacion.pdf

Pérez, F., Pérez , P., Nuñez-Comejo, P., Ibáñez, M., López, T., Juliá, C., . . . Ruiz de la Torre , R. (2013). Lumbalgia. En M. Belmonte, J. Castellano, J. Román, \& J. Rosas, Enfermedades Reumáticas. Actualización SVR. II Edición (págs. 741-768). Valencia, España: Sociedad Valenciana de Reumatología. Obtenido de https://svreumatologia.com/enfermedadesreumaticas-2/

Pérez, J. (2018). Incidencia de lumbalgia y factores asociados en pacientes adultos que acuden al Centro de Salud El Valle durante los meses de Julio 2017 a febrero 2018, Cuenca. Cuenca - Ecuador: Universidad de Cuenca, Facultad de Ciencias Médicas. Obtenido de http://dspace.ucuenca.edu.ec/bitstream/123456789/30230/1/PROYECTO\%20DE\%20IN VESTIGACI\%C3\%93N.pdf

Traeger, A., Buchbinder, R., Harris, I., \& Maher, C. (2017). Diagnosis and management of lowback pain in primary care. Canadian Medical Association Journal, 189(45), 1386-1395. doi:10.1503/cmaj.170527

Vlaeyen, J., Maher, C., Wiech, K., Zundert, J., Beraldo, C., Diatchenko, L., . . Linton , S. (13 de 12 de 2018). Low back pain. Nature Reviews Disease Primers, 4(52). doi:https://doi.org/10.1038/s41572-018-0052-1 